\title{
La presencia de criados particulares en la Casa de Dementes de Santa Isabel.
}

\author{
Encarnación Mollejo Aparicio. \\ Psiquiatra. Servicio de Salud Mental de Arganda del Rey. \\ Hospital Universitario del Sureste. Madrid. \\ encarnamollejo@wanadoo.es
}

La Casa de Dementes de Santa Isabel en Leganés $(1,2)$ surgió en el marco de la Ley de Beneficencia de 1849 (3), y el posterior Reglamento para su ejecución de 1852. A mediados del siglo XIX, Madrid carecía de un hospital psiquiátrico, solo disponía de una sala para 44 enajenados en el hospital general de Madrid (4). La Junta Provincial de Madrid acordó crear en Leganés un hospital para atender a los dementes, y fue inaugurado por Melchor Ordóñez en diciembre de 1851; para su construcción se utilizó una antigua casa que había pertenecido a los duques de Medinacelli $(5,6)$. Los primeros pacientes llegaron el $24 \mathrm{y}$ 25 de abril de 1852 procedentes del hospital general de Madrid (7), y por una real orden del 1 de noviembre de 1852, fue declarado manicomio nacional, recibiendo dementes de diferentes provincias $(8,9)$.

Los enfermos estaban distribuidos en tres categorías o clases, según el precio que pagaban por la estancia en el mismo; los gastos de los pobres corrían a cargo de la Beneficencia. En los primeros años y hasta 1868, los pensionistas de $1^{\mathrm{a}}$ clase pagaban 8 reales al día, y 4 reales los de $2^{\text {a }}$ clase o medio pensionistas. Según un documento encontrado en la historia $\mathrm{n}^{\circ} 262$, en1868 se incremento el precio de la estancia en el hospital, los pensionistas de $1^{\text {a }}$ clase pasaban de pagar 8 a 12 reales diarios, y los medio-pensionista pasaban de 4 a 6 reales al día. En el reglamento orgánico de 1873 continuaron mante- niéndose los 12 y 8 reales al día por estancia para los pensionistas de $1^{\mathrm{a}}$ y $2^{\mathrm{a}}$ clase respectivamente, además por el lavado y planchado de su ropa los pensionistas de ambas clases debían ingresar 10 reales al mes (10). La asistencia médica era la misma para todas las categorías, las diferencias se notaban en el alojamiento, el vestido y la comida. Los dormitorios, comedores y salas de recreo eran diferentes para cada clase; los pobres vestían con el traje que les proporcionaba el hospital, uno para verano y otro de invierno, sin embargo los pensionistas llevaban sus propias ropas y disfrutaban de algunas mejoras en las comidas, así a la ración habitual para todos los enfermos, ellos recibían postres, ensaladas y chocolate en el desayuno (10).

El reglamento de 1873 indicaba que el número de acogidos pensionistas de $1{ }^{\mathrm{a}}$ clase seria de 30 , de $2^{a}$ clase o medio pensionistas de 34 y de beneficencia 128 (10), sin embargo en las 2314 historias revisadas, el $53 \%$ de los pacientes ingresaron en la categoría de pensionistas. También he comprobado que algunos pacientes cambiaban de categoría durante su estancia, pasando a veces de pensionista a medio pensionista o pobre, cuando la familia no podía seguir pagando la cantidad estipulada.

Este trabajo es resultado del estudio realizado revisando y analizando 2.134 historias clínicas del Archivo Histórico del Manicomio de Santa Isabel de Leganés, de 
los pacientes ingresados entre 1852 y 1936 (11). El objetivo de este trabajo es presentar los documentos encontrados en las historias 239 y 256 de dos pacientes pensionistas, a quienes durante su ingreso en la Casa de Dementes de Santa Isabel en 1856, les acompañó un criado particular, perteneciente a sus familias, con el fin de servirles durante su estancia en el manicomio. Los documentos que presentamos son la solicitud de las familias pidiendo el ingreso del criado, junto con los informes del médico y director de la casa de dementes, en los que manifestaban los inconvenientes que habían encontrado en la experiencia con estos pacientes, y se oponían a aceptar un nuevo criado particular. Estos informes debieron servir de base para suprimir la entrada de criados particulares acompañando al enfermo durante su ingreso, pues son los únicos casos que he encontrado en las historias revisadas (11).

Desde la creación del hospital en 1852, el número de enfermos fue creciendo progresivamente llegando a superar los 200 en 1855 , la población manicomial se mantuvo en las dos centenas a lo largo del siglo XIX. El personal del manicomio en 1868 para atender a 218 pacientes era el siguiente: director, secretario-contador, administrador, comisario de entradas, auxiliar de arquitecto, un médico, dos practicantes, un capellán, 16 religiosas de la congregación de San Vicente de Paúl, un celador, un portero, un ordenan$\mathrm{za}$, un cochero, un hortelano, doce criados $\mathrm{y}$ ocho criadas (12).

Las religiosas Hijas de la Caridad de San Vicente de Paúl, se encargaron de la asistencia y cuidado de los pacientes desde la fundación de la Casa de Dementes, en los primeros años tuvieron una importante función en la administración, organización y economía interior de la casa (13).

Las funciones del personal del manicomio estaban recogidas en el reglamento orgánico de 1873 y $1885(10,14)$. Los criados se encargaban de cuidar y vigilar a los pacientes, de lavar y planchar la ropa, limpiar el establecimiento y preparar la comida (14). Según el art. 46 del reglamento de 1873, los criados cobraban 0,75 reales al día, más la ración de comida (10).

José María Miranda de la Paz fue el primer médico del hospital, nombrado por el gobernador de Madrid, Melchor Ordóñez el 23 de abril de 1852, otorgándole las plazas de médico titular de la villa de Leganés, y médico del hospital de dementes de Santa Isabel de Leganés, con la modesta retribución de 4.000 reales anuales, debiendo ejercer sus funciones de médico no sólo con los acogidos, sino también con los empleados del establecimiento (12). Según el reglamento de 1873, si el número de acogidos era inferior a 250 , solo habría un médico encargado de la asistencia, con un sueldo de 8.000 reales anuales y unas funciones definidas en los art. 77-83 del reglamento de 1873 (10). En el siglo XIX el número de acogidos nunca supero los $250 \mathrm{y}$ solo hubo un médico en el hospital, aunque el reglamento de 1885 indicaba que debía haber dos médicos: un jefe facultativo profesor de medicina, y un profesor agregado (14).

Durante los 22 años que José María Miranda ejerció de médico de la Casa de Dementes de Santa Isabel, se realizaron muchas de las importantes reformas y se establecieron las bases de la organización y el buen funcionamiento del manicomio. En 1855, redactó las primeras reglas higiénicas para el cuidado y asistencia de los enfermos del hospital de dementes de Santa Isabel, en ellas hacía referencia a la limpieza de la casa y de los asilados, los horarios para levantarse, los alimentos que debían tomar y, recomendaba que todos los días por la mañana y por la tarde, el practicante y una de las Hermanas de la Caridad pasaran revista general e individual de los acogidos, para comprobar que estaban 
limpios y aseados, prestando atención por si observaban algún signo o síntoma distinto de su enfermedad. José María Miranda realizó la primera memoria histórico-estadística de los tres primeros años del manicomio, aunque no se ha podido encontrar el original (12).

El dr. Miranda se encargo de formar y educar un cuerpo especial de enfermeros y criados para el cuidado de los alineados, aunque no se han conservado las reglas que dictó para ellos, se han encontrado referencias y comentarios a las mismas, en las que explicaba como el tratamiento de estos enfermos requería un mayor número de sirvientes, especialmente preparados. A través de Eduardo Viota, administrador depositario del manicomio, sabemos que cuando se intentó cambiar el servicio que el dr. Miranda había organizado, este se opuso, contestando de la siguiente forma: "El Art. 82 de la Instrucción General (1873), determina que en los hospitales haya un criado por cada diez acogidos. Nosotros contamos hoy con 203 asilados, 122 hombres y 81 mujeres, y nos corresponden precisamente los doce criados y ocho criadas que tenemos. $Y$ en nada puede reducirse este número, porque en el tratamiento médico que usamos con estos desgraciados, de acuerdo con los adelantos científicos, se hallan proscritos los castigos, los cerrojos y las ataduras, necesitándose observación exquisita, vigilancia permanente, ojos que no pierdan de vista un momento los movimientos desordenados y tumultuosos de estos seres ciegos, y manos que estén prontas a ayudarles y corregirles en sus peligrosas aberraciones.

La locura tiene diferentes fases y particulares exigencias, que tratada en hospitales, demanda mayor número de sirvientes especialmente instruidos y preparados. La asiduidad e insistencia que hay que emplear para el aseo y limpieza de estos enfermos, la abnegación y paciencia que hay que tener..., la táctica particular que hay que adquirir..., el peligro constante en que se vive..., son circunstancias que dificultan el servicio de los manicomios y que se deben remunerar para conseguirlas.

A fuerza de tiempo y enseñanza, hemos alcanzado el personal de criados que hoy tenemos y que llena nuestras aspiraciones. Procuremos conservarlos y no alterar la remuneración que hoy tienen, pues sólo así se secundará el generoso interés que por estos desdichados seres se toma el supremo gobierno de la nación" (12, pp. 45-46).

$\mathrm{Al}$ dr. Miranda se debe el proyecto de reglamento en 1858, que sirvió de base para los posteriores reglamentos de 1873 y 1885 . En ellos se reconocía la autoridad del médico en todo lo referente a la higiene, costumbres habituales, tratamiento ordinario o excepcional de los enfermos, debiéndole ayudar el practicante.

El médico ordenaba y disponía todo lo relativo al régimen físico y moral de los enajenados; supervisaba como se realizaba su vigilancia y limpieza, determinaba el grado de libertad interior y exterior que podían gozar, autorizaba o negaba las visitas de sus parientes, indicaba los castigos y las recompensas, proponía la clase de distracciones o trabajos que los enfermos podían disfrutar, certificaba sobre su estado de salud, determinando su curación y cuando podían salir de la casa. Sus obligaciones le imponían además la visita diaria a los enajenados, anunciándola con un toque de campana, y todos los pacientes debían esperarle a la entrada de los departamentos, para contestar a las preguntas que les hiciera.

Al médico le correspondía la redacción de los reglamentos interiores del asilo, señalando las obligaciones que competían a cada uno de los empleados en la asistencia de los enfermos. Era obligación del médico realizar la historia clínica y patogénica de cada en- 
fermo, anotando sus observaciones. También indicaba los casos en que debía procederse a la autopsia en las defunciones, y redactar cada año una memoria clínica y estadística, que debía presentar al vocal-visitador (15).

Pacientes que disfrutaron de un criado particular durante su ingreso en la Casa de Dementes Santa Isabel

El estudio de las historias clínicas como fuente documental resulta de una gran riqueza para la investigación historiográfica sobre la práctica y la asistencia psiquiátrica (16 - 18). Los documentos que presentamos, nos permiten obtener información sobre la actuación del médico, sus ideas sobre la enfermedad mental y sus reflexiones críticas al conceder un permiso de salida a un paciente, o negarse a aceptar la entrada de criados particulares en el hospital.

\section{Historia $239^{1}$}

D. José, soltero de 21 años, ingreso en la Casa de Dementes de Sta. Isabel de Leganés el 23 de abril de 1856, por padecer simplicidad de espíritu con alucinaciones. Ingresando en la categoría de pensionistas de primera clase.

Su padre el Marqués de Legarda al día siguiente del ingreso solicito al presidente de la junta general de Beneficencia, que acompañará a su hijo en el manicomio, un antiguo criado de la familia, a quien este respetaba y tenía en consideración. Aceptaron su petición el 29 de abril de 1856, debiendo pagar tres reales diarios por los gas-

\footnotetext{
1 Historia Clínica no 239, conservada en el Archivo Histórico del Manicomio de Santa Isabel de Leganés, que se encuentra en la biblioteca del actual Instituto Psiquiátrico Servicios de Salud Mental José Germain de Leganés.
}

tos de alimentación del criado, quedando este sujeto al director de la casa de dementes (el documento se puede leer a continuación del texto).

A los dos meses de su estancia en el manicomio su familia solicito un permiso para que el paciente pudiese salir del establecimiento y disfrutar de baños de mar. El médico del manicomio D. José Miranda informo favorablemente al director, sobre la conveniencia para el enfermo de este permiso aportando las siguientes razones:

"A pesar del poco tiempo que lleva este enfermo a nuestro cuidado, sus alucinaciones se han aliviado notablemente.... Es de tal naturaleza esta afección que se necesita mayor periodo de tiempo para conseguir resultados más largos y ventajosos.

El temperamento linfático de este paciente, la edad crítica en que se halla y su marcada predisposición al raquitismo y las escrófulas, indican que uno de los medios teóricos a su tratamiento son los baños de mar. Además vigilado y dirigido convenientemente, este enfermo encontrará más bien, que reducido y secuestrado en una casa de Dementes, debe de hacer mucho ejercicio, se le deben proporcionar continuas distracciones y ocupar consecuentemente su atención con objetos nuevos que le produzcan fuertes impresiones. Procede pues la concesión que solicita la familia del Sr..... de una licencia para llevar a tomar baños de mar".

Debido al informe favorable del médico, el paciente salió del manicomio el 30 de junio de 1856 con un permiso para disfrutar de baños de mar y después no volvió a incorporarse al hospital.

El único tratamiento que recibió este paciente durante su ingreso fue el internamiento psiquiátrico. De los 1.221 pacientes ingresados entre 1852 y 1899 en Leganés, solo el $19 \%$ de ellos recibieron un tratamiento complementario al ingreso psiquiátrico. 
El medio más empleado en este periodo fue la hidroterapia, aplicada en distintas modalidades como baños o duchas, solos o combinados con agua a distintas temperaturas, $\mathrm{y}$ con diferente duración. Existían también los baños generales con sales marinas o también llamados baños de mar artificiales, en los que se añadían sustancias al agua para favorecer los efectos sedantes del baño (19).

Los enfermos podían disfrutar de salidas temporales o permisos durante la estancia en el manicomio, estas se realizaban a petición de la familia o por indicación del médico como prescripción exploradora previa al alta definitiva del enfermo, o como tratamiento coadyuvante. En todos los casos y previo a la salida temporal o definitiva del hospital, el médico debía informar de la conveniencia o no de la misma. Si la licencia no excedía de 2 meses, se conservaba la plaza del enfermo hasta su vuelta (19).

\section{Historia $256^{2}$}

D. Fernando de 32 años y de profesión abogado, ingresó en la Casa de Dementes de Leganés en junio de 1856 como pensionista de $1^{\text {a }}$ clase, con el diagnóstico de manía crónica, permaneció ingresado durante 8 años, hasta su muerte en el hospital por una afección tuberculosa en junio de 1864. Era habitual que los paciente permanecieran en el manicomio hasta el final de sus vidas, así de los 2.314 pacientes ingresados entre 1852 y 1936, el $65 \%$ fallecieron durante su ingreso en el hospital, siendo las causas más

\footnotetext{
2 Corresponde a la historia clínica 256, del archivo de historias clínicas del Hospital de Santa Isabel de Leganés, que se encuentra en la biblioteca del actual Instituto Psiquiátrico Servicios de Salud Mental “José Germain" de Leganés.
}

frecuentes de defunción las de origen respiratorio (20). La estancia media de los pacientes ingresados en el manicomio de Leganés en ese periodo fue de 8 años, aunque algunos enfermos llegaron a estar 40, 50 y hasta 57 años en el manicomio (19).

El diagnóstico más frecuente de los pacientes ingresados hasta 1936 fue manía en sus variadas formas clínicas, seguido de trastornos esquizofrénicos, englobando en esta categoría la demencia precoz, paranoia, parafrenia, hebefrenia y psicosis (21). Entre 1852 y 1881 predominó el diagnóstico de manía, después disminuyo de forma progresiva coincidiendo con la aparición de nuevas entidades nosológicas para definir las enfermedades mentales (19).

En la historia 256 se encuentran cartas de la familia, solicitando la admisión de un criado procedente de su casa, para atender al enfermo en el hospital. La primera carta es del 9 julio de 1856 (esta carta se incluye a continuación del texto), quedando reflejado en la historia que fue admitido inmediatamente, pagando la familia tres reales diarios por la estancia del criado en el manicomio.

Según las anotaciones de la historia clínica, el criado particular de D. Fernando fue despedido del hospital, pero no se especifica la fecha, ni el motivo de su expulsión. Esta firmado por Faustino Mesa comisario de entradas, después fue secretario contador en 1858, y director del establecimiento entre 1859 y 1862 (12).

En mayo de 1858, la madre del paciente volvió a solicitar que se admitiera en el hospital, un criado contratado por ella para el cuidado de su hijo, sin embargo en está ocasión, no se acepto su petición, por los informes desfavorables que emitieron el médico del hospital y el director, donde expresaban las razones por las que desaconsejaban esta práctica y manifestaban su negativa a admitir criados particulares en el hospital. 
Informe del médico $\left(H^{a} 256\right)$.

El informe del 26 de mayo de 1858, de José Maria Miranda médico del hospital, exponía las razones por las que no se debía acceder a la solicitud para admitir un criado particular para el servicio del acogido D. Fernando (reproducimos su informe al final del texto). El dr. Miranda justificaba y razonaba su negativa a la utilización de criados particulares pagados por la familia para el servicio de los enfermos, exponiendo una serie de motivos generales en los que hacia referencia a que está misma práctica en otras casas de dementes del extranjero se había considerado negativa, aludía a la experiencia previa con dos criados particulares en la casa, señalaba que con estos criados no se conseguía el deseado aislamiento del paciente, considerado uno de los principios terapéuticos básicos de la época para la enfermedad mental, además no se tenia sobre estos criados la misma autoridad que con los otros contratados por el hospital, eran un mal ejemplo para los otros sirvientes y despertaban envidias en algunos enfermos, y en el caso concreto de D. Fernando, no suponía ningún beneficio para su curación, por la patología y características del sujeto.

Durante el tiempo que permaneció en el hospital, el único tratamiento que recibió este paciente fue el internamiento psiquiátrico, en el siglo XIX era considerado el método terapéutico por excelencia. El primer alienista para quien la institucionalización de los enfermos conllevaba beneficios terapéuticos fue William Battie (1703-1776), fundador del Hospital de St. Luke en Londres. En 1758 escribió Treatise on Madness, en el cual atribuía virtudes terapéuticas al manicomio, decía: "el manejo de los pacientes hacia más que las medicinas; y la experiencia repetida me ha convencido de que el confinamiento solo es a menudo suficiente, y siempre tan necesario, que sin el, cualquier método hasta ahora inventado para la curación de la locura seria inefectivo" (22, p.68). Battie recomendaba como cura el aislamiento, los pacientes no debían recibir visitas de amigos, ni ser atendidos por sus propios sirvientes, sino por los empleados del manicomio.

Voison también consideraba el aislamiento, la primera medida terapéutica, decía: "De todos los medios que la medicina puede aportar a la terapéutica de las enfermedades mentales yo no conozco ninguno más poderoso que el aislamiento; su influencia sobre el cerebro es a la vez activa y pasiva" (23, p.392).

En 1861, Manuel Mesa escribía sobre los tratamientos aplicados en Santa Isabel, "El medio terapéutico que primeramente debe usarse y el cual produce resultados muy felices, es el aislamiento, entendiéndose por tal, el cambio completo de todos los objetos que rodean al enfermo; aislamiento que debe llevarse en ocasiones hasta el extremo de no dejarles ver a sus parientes y demás personas con quien estaba acostumbrado a vivir" (24, p.396)

Se creía que todo lo que atañía a las representaciones anteriores del enfermo excitaba de nuevo sus ideas morbosas, y contribuía a fijarle en su delirio. Había que despertar en el enfermo otras sensaciones, e interrumpir las cadenas anteriores de pensamiento. Por este motivo se suprimían las visitas de familiares y amigos, para evitar una agravación de la enfermedad. También se recomendaba dar al enfermo ropa diferente, instalarle en una habitación desconocida y que lo vigilaran personas extrañas, incluso quitarle lo que tuviera en los bolsillos que pudiera recordarle su situación anterior (25).

En el siglo XIX, el manicomio se consideraba el principal medio terapéutico, realizando el aislamiento del paciente de su ambiente, considerado patógeno y nocivo 
para él, además permitía aplicar los diversos medios terapéuticos disponibles en ese momento (26).

\section{Informe del director}

Desde su fundación en 1852, la dirección del hospital fue fundamentalmente de carácter administrativo e independiente de la jefatura facultativa ejercida por el médico. Las funciones del director estaban recogidas en el proyecto de reglamento de 1858 de Gómez de la Serna, y en el reglamento orgánico e interior del Hospital de Santa Isabel de Leganés de 1873, en el capítulo X, artículos 47-49 (10).

El director era responsable de cuanto ocurriera dentro del recinto del manicomio, pudiendo tomar en el acto las medidas urgentes que la situación reclamará, a él le estaban subordinados todos los empleados de la casa, todas las cuentas del hospital eran supervisadas por él y tenía la facultad de proponer reformas, la adquisición de material mobiliario, facultativo, ropas y toda clase de repuestos según las necesidades del manicomio, poniéndolo en conocimiento de la junta general para su aprobación. Debía informar de las solicitudes de licencia temporal, presentadas por una persona en representación del asilado, acompañado del dictamen del médico del hospital. Informaba a la autoridad correspondiente, de la curación de un demente ingresado por disposición de los tribunales de justicia; demandaba al alcalde de Leganés la hoja de ruta para trasladar a un enfermo curado a su domicilio, cuando después de habérselo comunicado a la familia, esta no acudía a recogerlo en el plazo de 90 días; ordenaba que se cumpliera todo lo dispuesto por el médico sobre el tratamiento físico-moral, la higiene y seguridad de los enfermos; determinaba las horas de apertura y cierre de las puertas del establecimiento, teniendo en su poder las llaves o bien entregándolas a un empleado de su confianza (15).

El informe del 27 de mayo de 1858, de Manuel González director del hospital (el informe está incluido en el texto), dirigido al presidente de la Junta General de Beneficencia, manifestaba los inconvenientes de aceptar la solicitud de Ana $\mathrm{M}^{\mathrm{a}}$ sobre la admisión de un criado particular para el cuidado de su hijo, indicando que alteraba y perjudicaba el funcionamiento de la casa, al provocar envidias en los otros sirvientes que tenían que atender mayor número de pacientes, además al estar a las órdenes directas de la familia, a veces debía salir de la casa para hacerles recados o encargos, dejando al enfermo solo, sin que se ocupasen de él los otros criados de la casa, bien porque no sabían que estaba solo, o porque no lo consideraban de su competencia; tampoco consideraba que fuera provechoso para el acogido la asistencia permanente de un criado particular. El director coincidía en sus opiniones y criterios con los del médico del establecimiento, sobre los inconvenientes que conllevaba la presencia de criados particulares en el manicomio, por lo que no se volvió a aceptar la entrada de los mismos para el servicio y cuidado de los enfermos, manteniéndose este criterio en los años posteriores.

\section{BIBLIOGRAFÍA:}

(1) Delgado M. Los veinte primeros años del Manicomio Modelo de Leganés (1852-1871), Asclepio, 1986; 38: 273-297.

(2) Peset JL. El manicomio modelo en España. En: VV.AA., Un siglo de Psiquiatría en España, Madrid: Extraeditorial; 1995. p. 43-51. 
(3) Hernández Iglesias F. La Beneficencia en España. Madrid: Establecimiento Tipográfico de Manuel Minuesa; 1876.

(4) Núñez Olarte JM. El Hospital General de Madrid en el siglo XVIII. (actividad médico-quirúrgica). Madrid: CSIC; 1999.

(5) Villasante O. El manicomio de Leganés. Debates científicos y administrativos en torno a un proyecto frustrado. Rev. Asoc. Esp. Neuropsiq. 1999; 19, (71): 469479;

(6) Villasante O. The unfulfilled project of the Model Mental Hospital in Spain: fifty years of the Santa Isabel Madhouse, Leganés (1851-1900). History of Psychiatry, 2003; 14, (1): 3-23.

(7) Memoria del Hospital Provincial de Madrid. Madrid: Oficina Tipográfica; 1875, p. 99-102

(8) Salas y Vaca J. Mejoras realizadas en los servicios del Manicomio Nacional. Madrid: Est. Tip. Artes Gráficas; 1929.

(9) Tierno R. Demografía psiquiátrica y movimientos de la población del Manicomio Nacional de Santa Isabel (19311952). Frenia 2008; 8: 97-130.

(10) Reglamento Orgánico e Interior del Manicomio de Santa Isabel de Leganés. En: Instrucción general y reglamentos interiores de asilos y colegios pertenecientes a la beneficencia general. Madrid: Imprenta Nacional; 1873.

(11) Mollejo Aparicio E. Evolución de los criterios diagnósticos y terapéuticos en el Hospital Psiquiátrico de Leganés (18561936). [Tesis doctoral] Facultad de Medicina. Universidad de Salamanca; 2001.

(12) Viota y Soliva E. Memoria Histórica del Hospital de Dementes de Santa Isabel de Leganés. Madrid: Est. Tipográfico de A. Avrial; 1896.

(13) Pérez de Guzmán y Gallo P. Memorial de la vida de Sor Teresa Viver y Can- dell. Superiora de las Hijas de la Caridad del Hospital de Dementes de Santa Isabel de Leganés, único de la Beneficencia en España. Madrid, Est. Tipográfico de Fortanet. 1911.

(14) Reglamento Orgánico e Interior del Manicomio de Santa Isabel de Leganés. Gaceta de Madrid, 15 de Mayo de 1885, 135; (2): 447-450.

(15) Mollejo Aparicio E. Funcionamiento y régimen interno del manicomio de Santa Isabel de Leganés (1852-1936). En: Fuentenebro F, Huertas R. (Editores) Historia de la psiquiatría en Europa. Madrid: Frenia; 2003. p. 639-646.

(16) Huertas García-Alejo R. Las historias clínicas como fuente para la historia de la psiquiatría: posibles acercamientos metodológicos, Frenia 2001; 1 (2): 7-38.

(17) Livianos Aldana L. La historia clínica como fuente para la historia de la psiquiatría. En: Fuentenebro F. y Huertas R. (editores) Historia de la psiquiatría en Europa, Madrid: Frenia; 2003. p.773-784.

(18) Laín Entralgo, P. La historia clínica. Historia y teoría del relato patográfico, Madrid: Triacastela; 1998.

(19) Mollejo Aparicio E. Historia del Manicomio de Santa Isabel. Evolución de los diagnósticos y tratamientos de 1852 a 1936. Madrid: Colegio Oficial de Médicos de Madrid; 2011.

(20) Mollejo Aparicio E, del Cura González M, Huertas García-Alejo R. La práctica clínica en el Manicomio de Leganés (1852-1936). Primera aproximación al estudio de sus historias clínicas. En: Martínez Pérez J. (comp.). La medicina ante el nuevo milenio: una perspectiva histórica. Cuenca: Universidad de Castilla- La Mancha, 2004, p. 149-162.

(21) Mollejo Aparicio E. Evolución del diagnóstico de esquizofrenia en el Manicomio de Leganés (1952-1936). En: Martínez Pérez J, Estévez J, del Cura M, Blas L. 
(eds.) La gestión de la locura: conocimiento, prácticas y escenarios (España, siglos XIXXX). Cuenca: Universidad de Castilla- La Mancha; 2008, p. 85-104.

(22) Battie W. A Treatise on Madness. Londres: Whiston; 1758.

(23) Voison F. Des causes morales et psysiques des maladies mentales. París: 1826.

(24) Mesa M. La casa de dementes de Santa Isabel de Leganés. La España Médica. 1861; 6: 363-366 y 395-397.
(25) Kraepelin E. Cien años de Psiquiatría. Madrid: Asoc. Esp. Neuropsiq; 1999.

(26) Crespillo Calleja JM. El fármaco como alternativa terapéutica de la enfermedad mental en el Hospital Psiquiátrico Santa Isabel de Leganés, durante su primer siglo de actividad asistencial 1852-1951. [Tesis doctoral] Facultad de Farmacia de la Universidad de Alcalá de Henares. 1986 
434

E. Mollejo

HISTORIAS CON HISTORIA

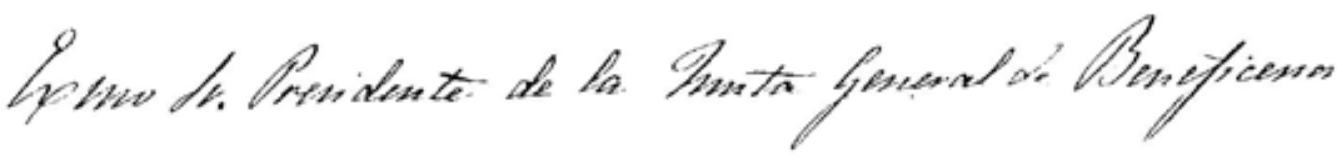

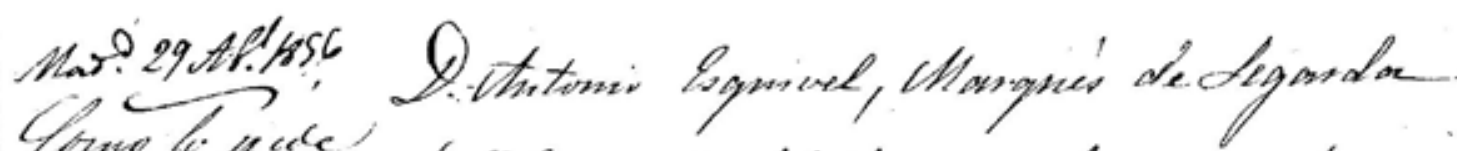

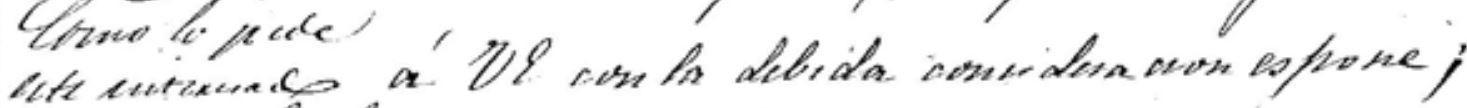

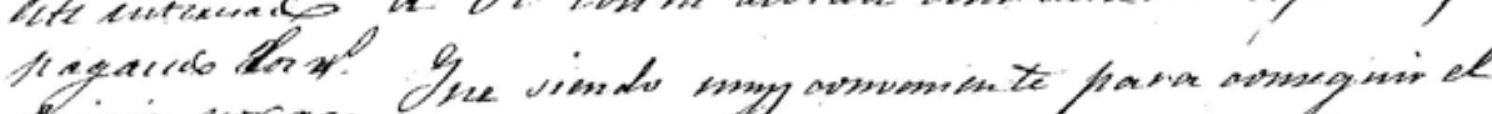

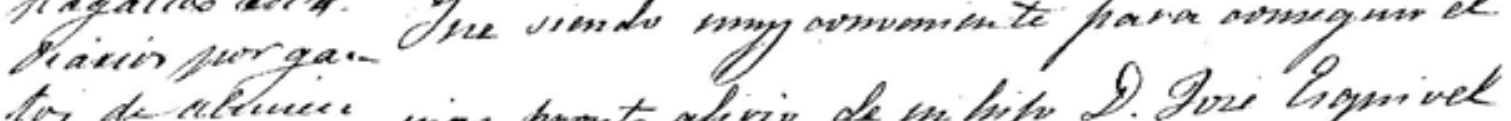

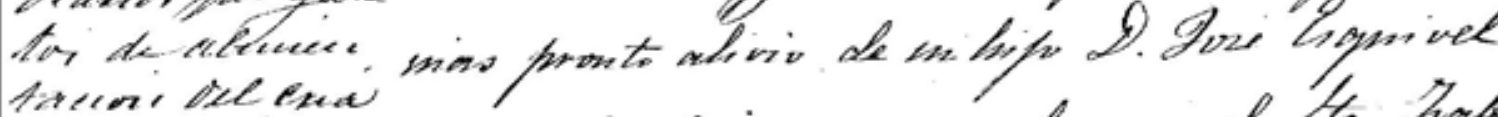

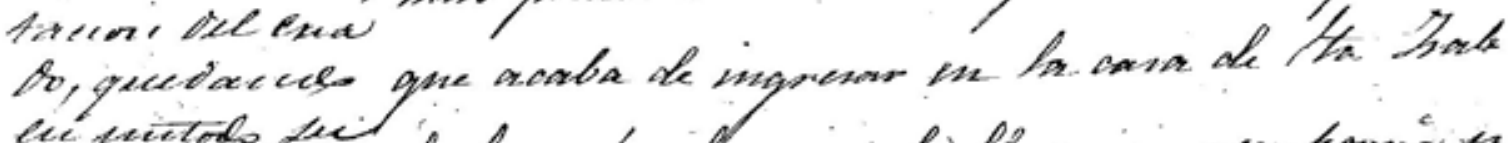

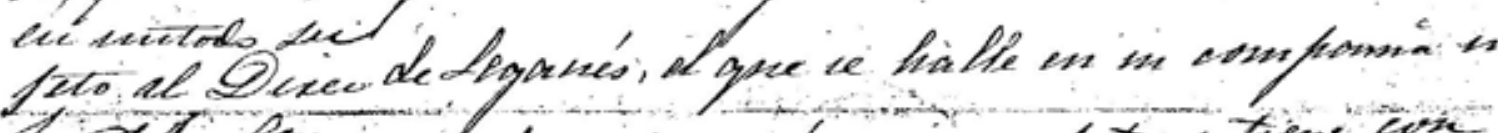

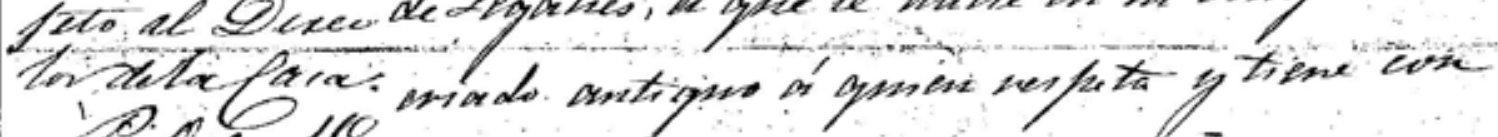

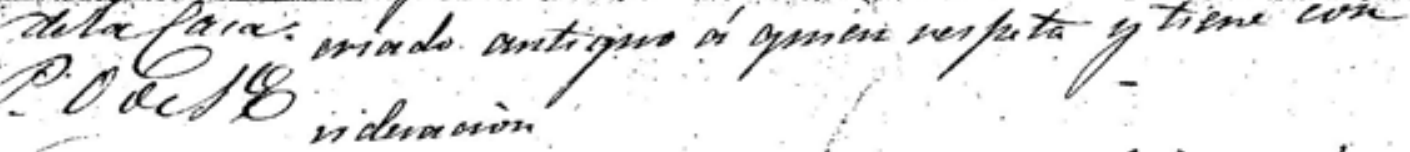

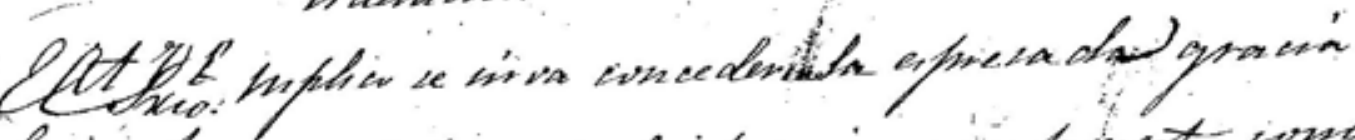

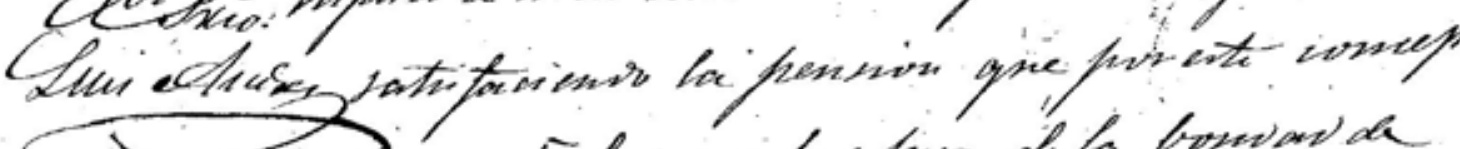

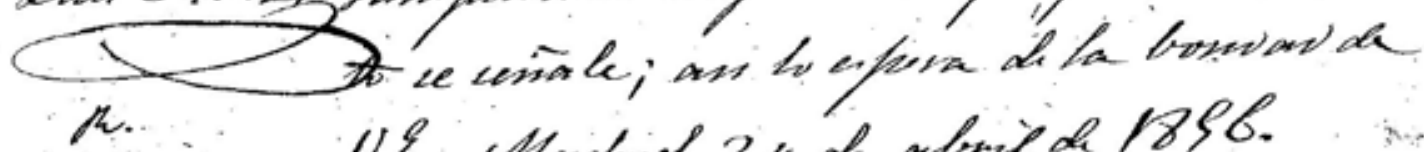
U9. Anminid 24 de abried 1896.

Elonong desegoridas 
Excmo. Sr. Presidente de la Junta General de Beneficencia

D. Antonio Esquivel, Marqués de Legarda a V.E. con la debida consideración expone:

Que siendo muy conveniente para conseguir el más pronto alivio de su hijo D. José Esquivel que acaba de ingresar en la casa de Sta. Isabel de Leganés, el que se halle en su compañía un criado antiguo a quien respeta y tiene en consideración.

Suplico se sirva conceder la expresada gracia, satisfaciendo la pensión que por este concepto se señale; así lo espera de la bondad de V.E. Madrid 24 de abril de 1856.

El Marqués de Legarda

(Anotación en el margen izq., de la hoja)

Madrid 29 abril 1856

Como 10 pide este internado pagando tres reales diarios por gastos de alimentación del criado, quedando en todo sujeto al director de la casa.

P.O. de S.E.

El Secretario

Luís Andrés

Historia 239. Petición de la familia de dos pacientes, solicitando al Presidente de la Junta General de Beneficencia, el ingreso de un criado particular en la Casa de Dementes de Santa Isabel de Leganés. (Archivo Histórico del Manicomio de Santa Isabel de Leganés, biblioteca del Instituto Psiquiátrico, Servicios de Salud Mental José Germain de Leganés). 
436

E. Mollejo

HISTORIAS CON HISTORIA

2.2. .5.5.

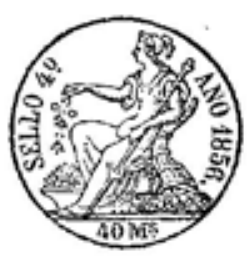

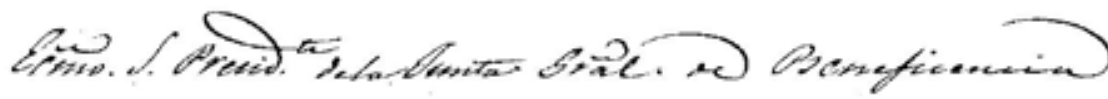

9. de Vielis 1854

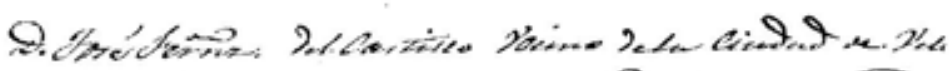

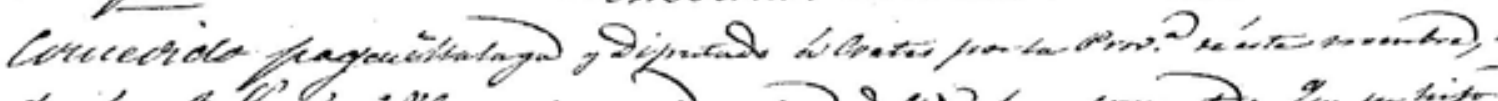

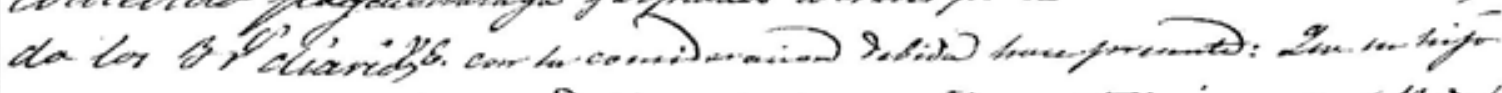

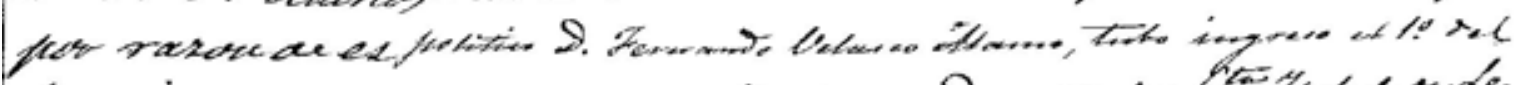

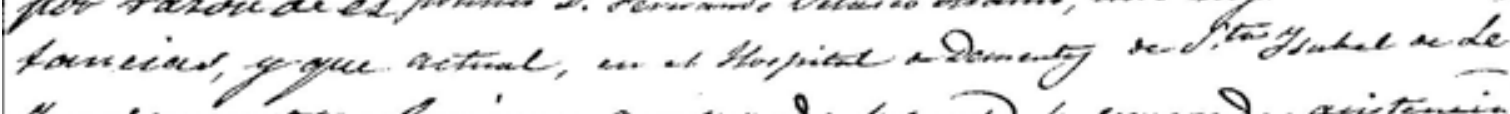

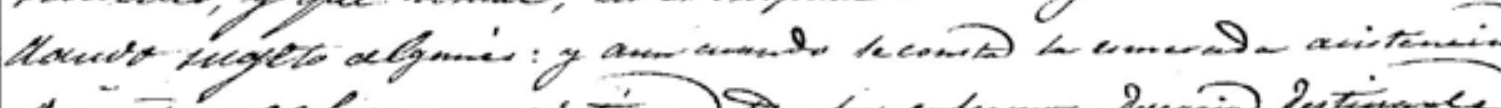

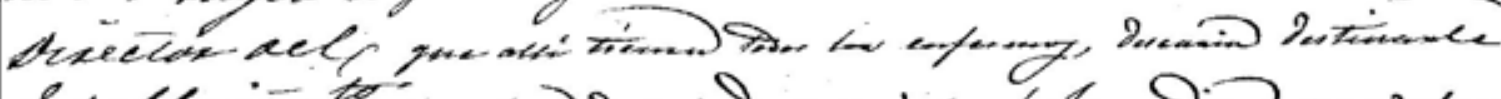

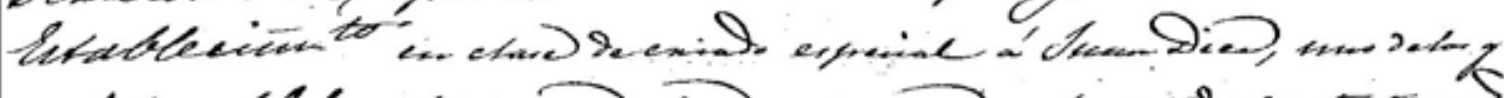
dodet.

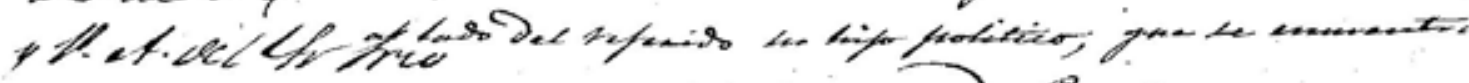

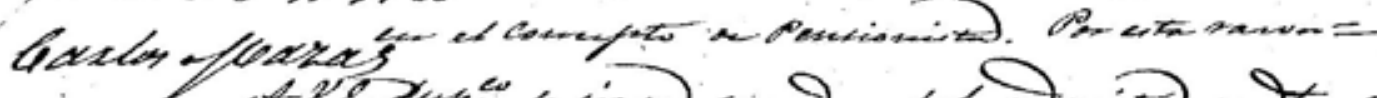

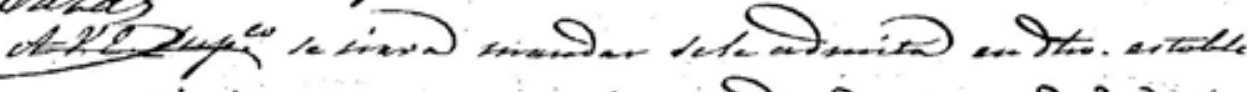

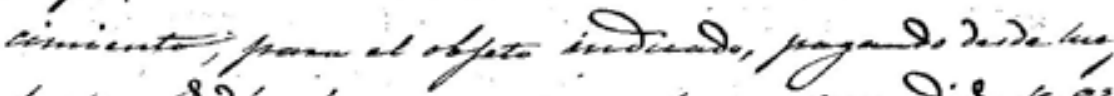

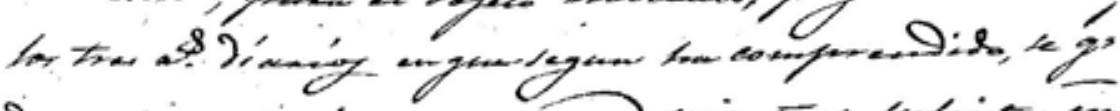

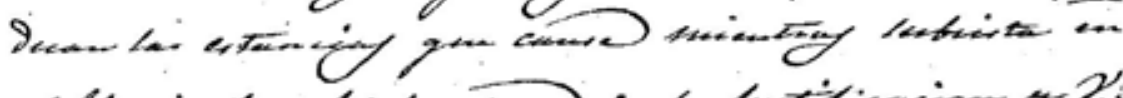

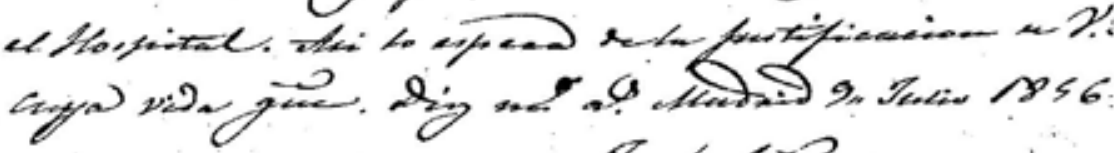
Fó dixis: recontizhs 
HISTORIAS CON HISTORIA

Excmo. S. Presidente de la Junta Gral. de Beneficencia

D. José Fernández del Castillo vecino de la ciudad de Vélez, Málaga y diputado de las Cortes por la provincia de ese nombre.

V. E. Con la consideración debida hace presente: Que su hijo político D. Fernando Velasco Álamo, tuvo ingreso el $1^{\circ}$ del actual, en el Hospital de Dementes de Sta. Isabel de Leganés: y aun cuando me consta la esmerada asistencia que allí tienen todos los enfermos, desearía destinase en clase de criado especial a Juan Diez, uno de los que el exponente tiene en su casa, y ha estado constantemente al lado del referido su hijo político, que se encuentra en el concepto de pensionista. Por esta razón $=$

Suplico se sirva mandar se le admita en vuestro establecimiento, para el objeto indicado, pagando desde hoy los tres reales diarios en que según he comprendido, se gradúan las estancias que cause mientras subsista en el Hospital. Así lo espera de la justificación en $\mathrm{V}$.

Cuya vida guarde Dios m. a. Madrid 9 julio 1856

José Fernández del Castillo

(Anotación del margen izq., en la misma hoja) 9 de julio 1856

Concedido pagando los tres reales diarios por razón de estancias, y quedando sujeto al director del establecimiento.

D.V. de S. E.

y V.A. del Sr. Secretario Carlos Plazas.

A V.E.

Historia 256. (Archivo Histórico del Manicomio de Santa Isabel de Leganés, biblioteca del Instituto Psiquiátrico, Servicios de Salud Mental José Germain de Leganés). 
GHW

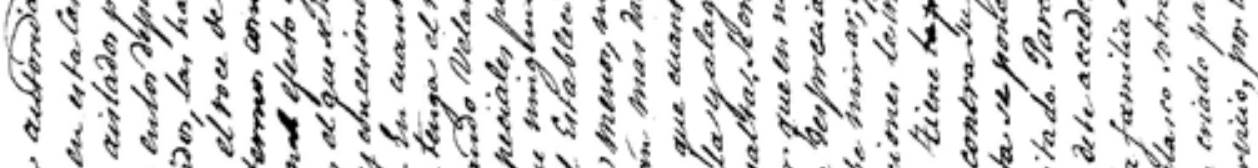

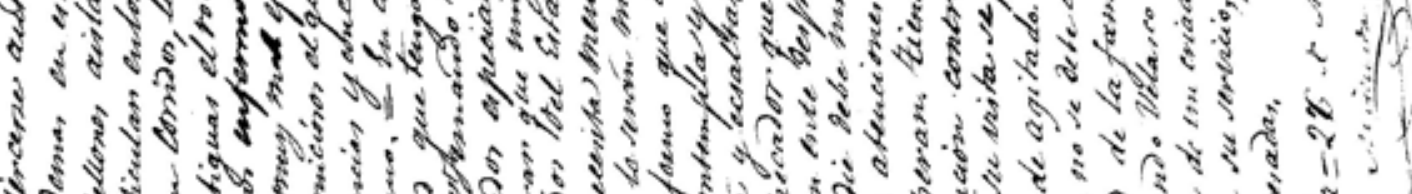

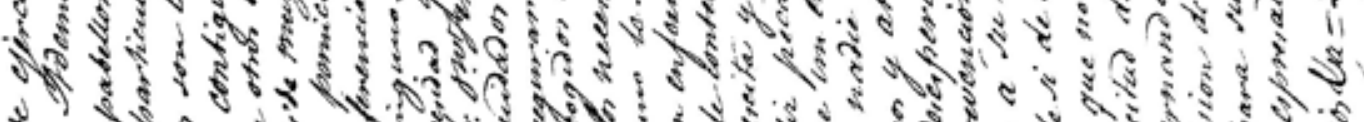

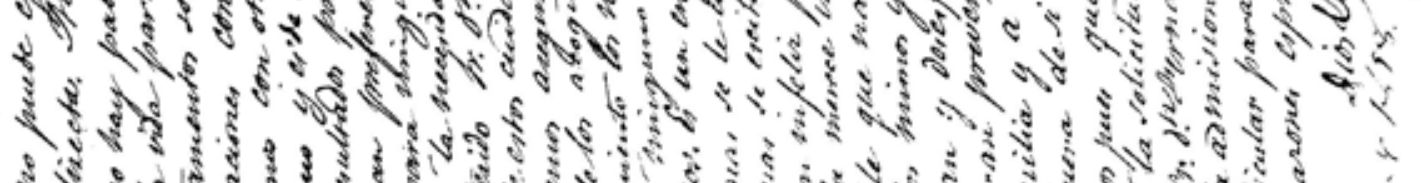

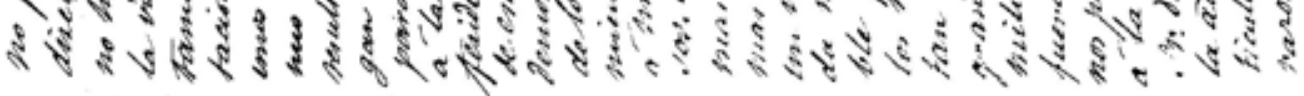

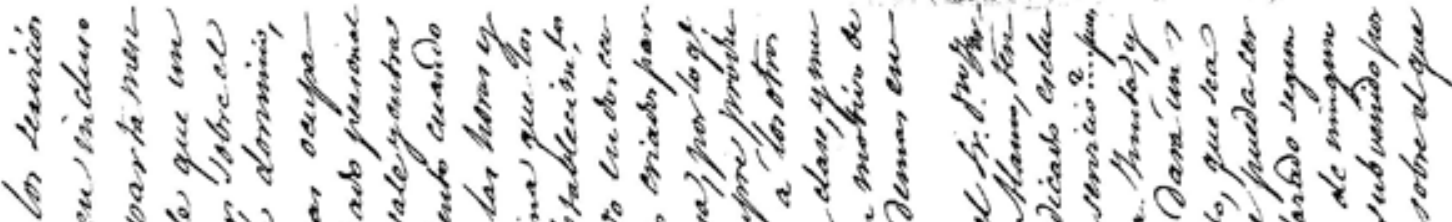

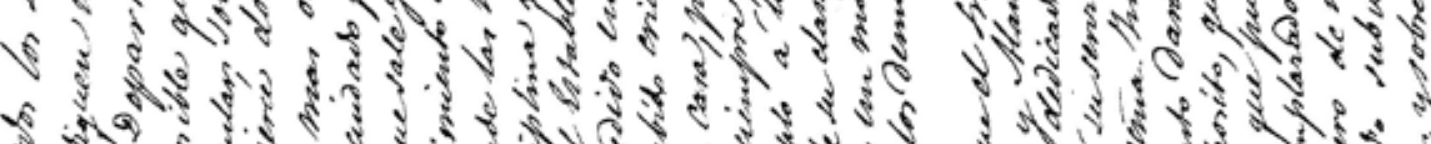

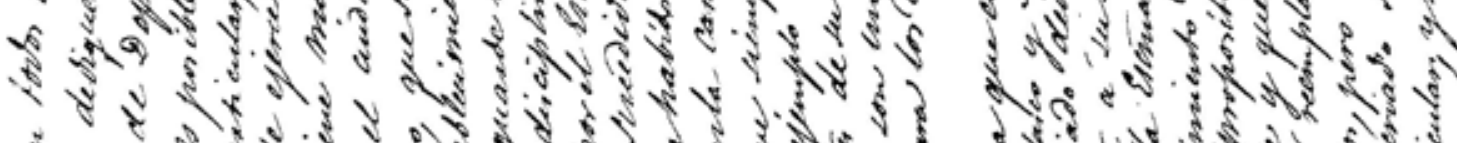
6. 3. no

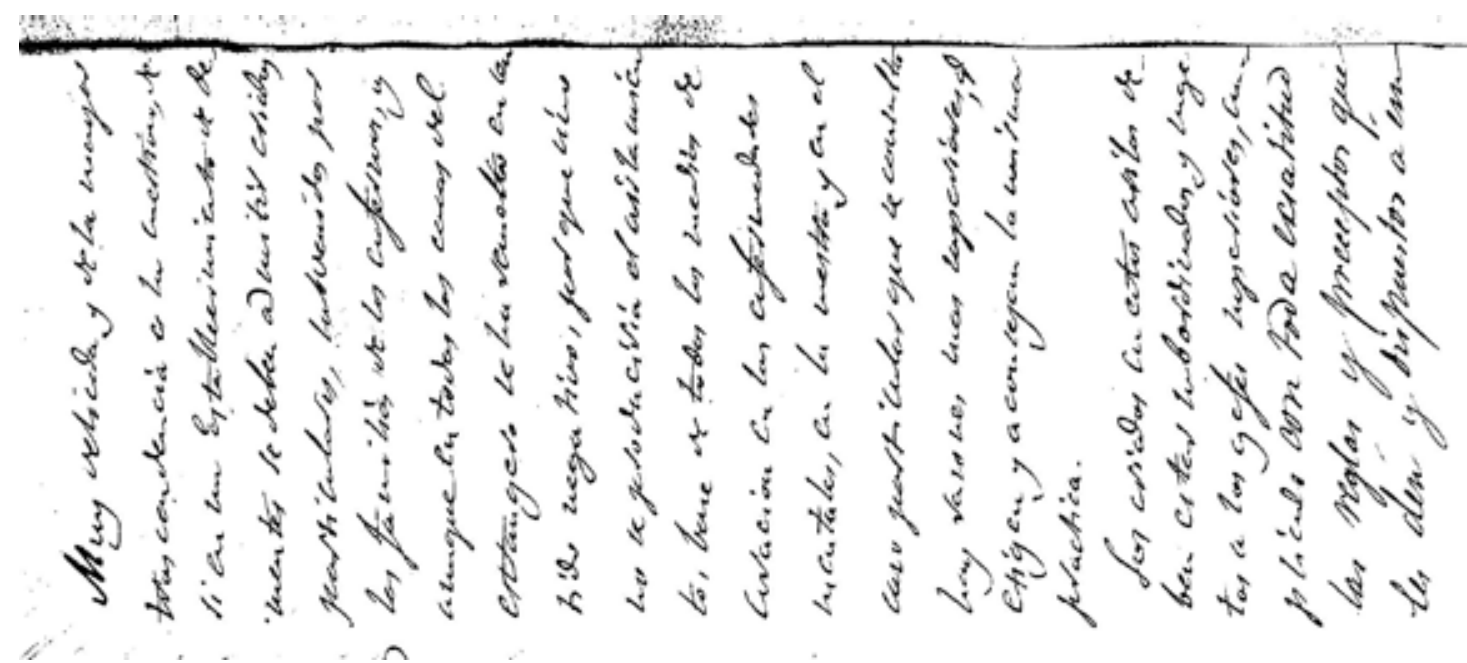


Muy delicada y de la mayor trascendencia es la cuestión, de sí en un establecimiento de dementes se deben admitir criados particulares, subvenidos por las familias de los enfermos, y aunque en todas las casas del extranjero se ha resuelto en sentido negativo, porque sino no se produciría el aislamiento, base de todos los medios de curación de las enfermedades mentales, en la nuestra y en el caso particular que se consulta hay razones más especiales, que exigen y aconsejan la misma práctica.

Los criados en estos asilos deben estar subordinados y sujetos a los jefes superiores, cumpliendo con toda exactitud las reglas y preceptos que les den y dispuestos a emplearse en todos los servicios a que los dediquen incluso al cambio de departamento, y no es posible con un criado particular, sobre el que no se ejerce dominio, que no tiene más ocupación, que el cuidado personal de su amo, que sale y entra en el establecimiento cuando le place, guarde las horas y tenga la disciplina que los pagados por el establecimiento. Esto ha sucedido en dos casos que ha habido criados particulares en la casa, por lo que creemos, que siempre producen mal ejemplo a los otros dependientes de su clase, y muchas veces son un motivo de envidia para los demás enfermos.

¿Si desea que el Sr. D. Fernando Velasco y Alamo, tenga un criado dedicado exclusivamente a su servicio?, pues pídase a la Excma. Junta, y el establecimiento dará un criado a propósito, que sea responsable y que pueda ser removido o reemplazado según sus servicios, pero de ningún modo un criado subvenido por un particular, y sobre el que no puede ejercerse autoridad directa. Además en esta casa no hay pabellones aislados para la vida particular, los departamentos son corridos, las habitaciones contiguas, el roce de unos con otros enfermos continuo y es de muy mal efecto y resultados perniciosos el que se hagan preferencias y concesiones para ninguno. = En cuanto a la necesidad que tenga el referido Sr. D. Fernando Velasco de estos cuidados especiales podemos asegurar que ninguno de los acogidos del establecimiento los necesita menos, ni a ninguno le serán más dañosos. Es un enfermo que cuanto más se le contempla y alaga (sic), más se excita y exalta. Se cree un infeliz pecador que en nada merece, un ente despreciable, que nadie debe mimar; y los mimos y atenciones le irritan y desesperan. Tiene gran prevención contra su familia y a su vista se pone fuera de sí de agitado. Parécenos pues que no se debe acceder a la solicitud de la familia del Sr. D. Fernando Velasco sobre la admisión de un criado particular para su servicio, por las razones expresadas.

Dios guarde a Ud. $=26$ de mayo 1858 Miranda .

Historia 256. Informe del médico José $\mathrm{M}^{\mathrm{a}}$ Miranda (Archivo Histórico del Manicomio de Santa Isabel de Leganés, biblioteca del Instituto Psiquiátrico, Servicios de Salud Mental José Germain de Leganés). 


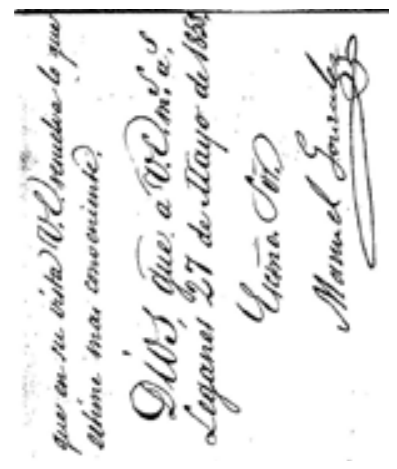

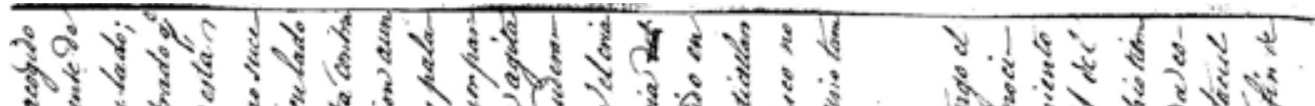
MHMHMU M M H

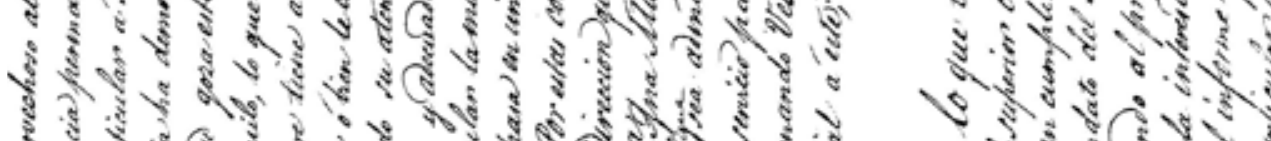

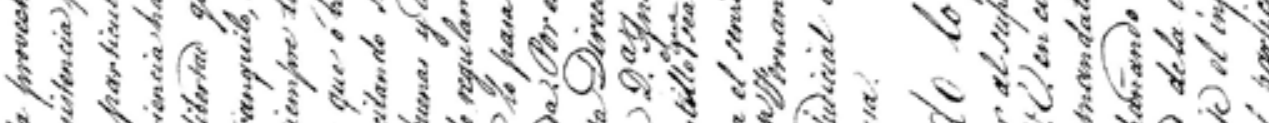

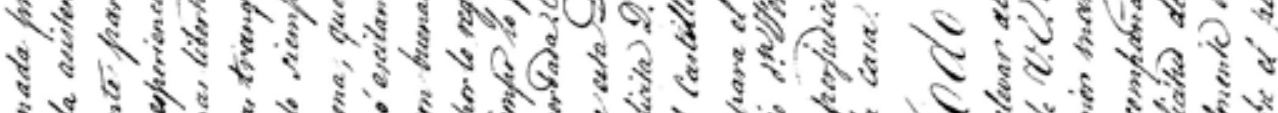

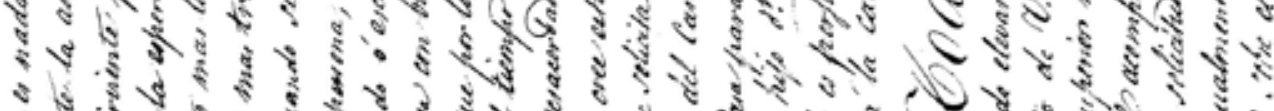

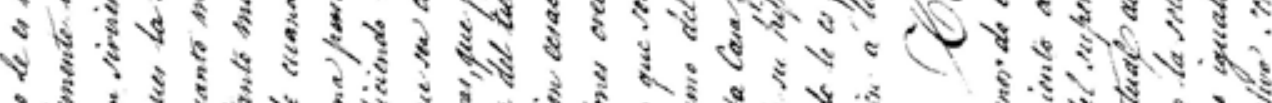

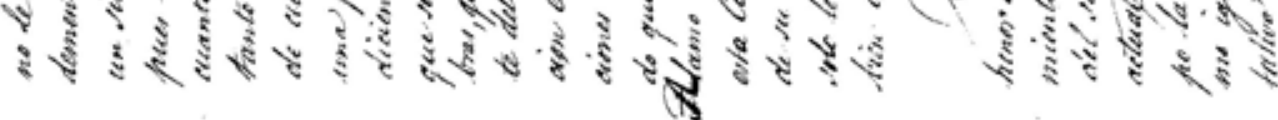

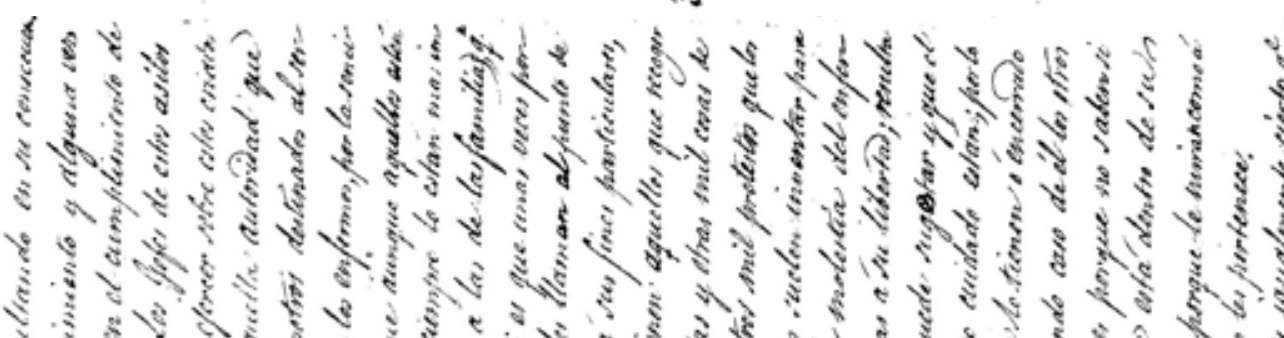

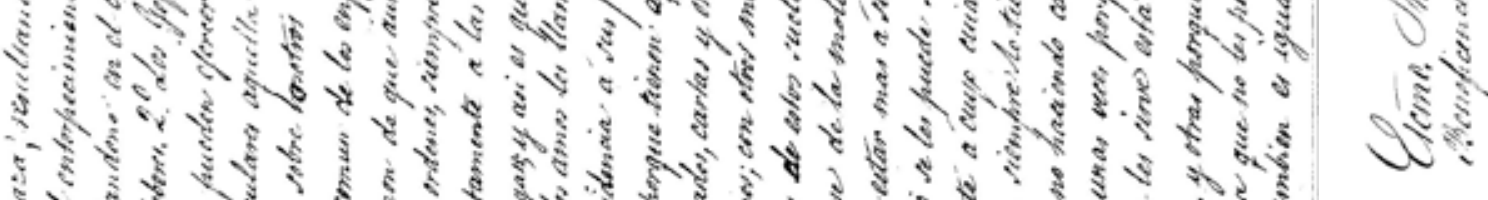

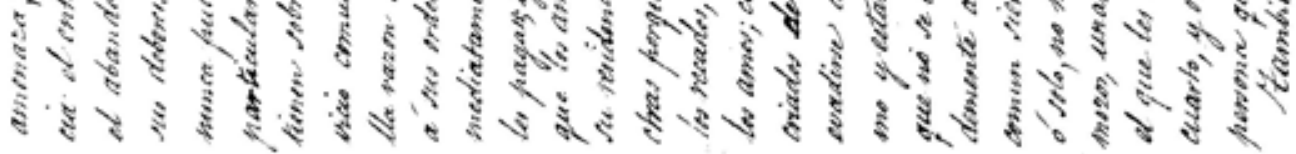

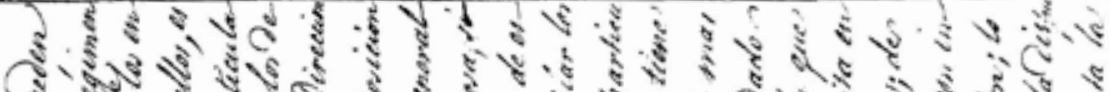

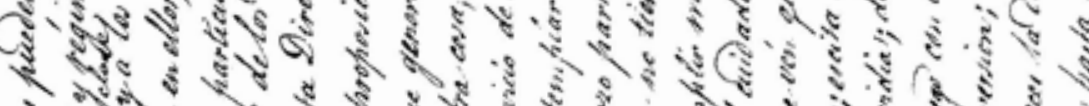
jo

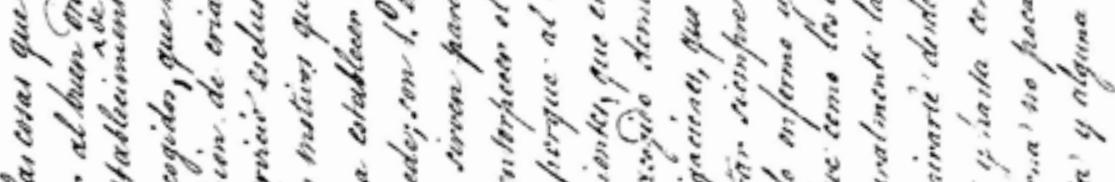

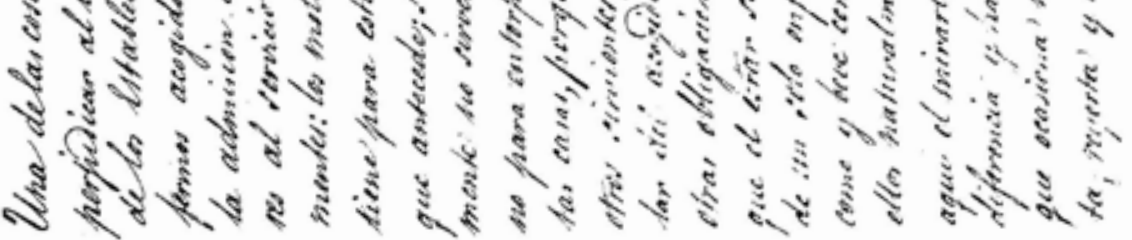




\section{Excmo. Sr.}

Una de las cosas que más pueden perjudicar al buen orden y régimen de los establecimientos de esta clase, y a los enfermos acogidos, que hay en ellos, es la admisión de criados particulares al servicio exclusivo de los dementes: los motivos que esta Dirección tiene para establecer la proposición que antecede; son $1^{0}$ el que generalmente no sirven para otra cosa, sino para entorpecer el servicio de estas casas, porque al contemplar los otros sirvientes, que el mozo particular del acogido demente no tiene otras obligaciones que cumplir, más que el estar siempre al cuidado de un solo enfermo y que ven que come y beve ( $\mathrm{sic}$ ) como los otros, excita en ellos naturalmente la envidia; de aquí él mirarle desde luego con indiferencia y hasta con aversión; lo que ocasiona no pocas veces la disputa, reyerta y alguna vez la amenaza; resultando en su consecuencia el entorpecimiento y alguna vez el abandono en el cumplimiento de sus deberes. $2^{\circ}$. Los jefes de estos asilos nunca pueden ejercer sobre estos criados particulares aquella autoridad que tienen sobre los otros destinados al servicio común de los enfermos, por la sencilla razón de que aunque aquellos estén a sus ordenes, siempre lo están más inmediatamente a las de las familias que les pagan, y así es que unas veces porque los amos les llaman al punto de su residencia a sus fines particulares, otras porque tienen aquellos que recoger los recados, cartas y otras mil cosas de los amos; con otros mil pretextos que los criados de estos suelen inventar para evadirse de la molestia del enfermo y estar más a su libertad; resulta que no se les puede sugetar (sic) y que el demente a cuyo cuidado están; por lo común siempre lo tienen o encerrado ó solo, no haciendo caso de él los otros mozos, unas veces porque no saben si el que los sirve está dentro de su cuarto, y otras porque le miran como a persona que no les pertenece.

También es igualmente cierto que no le es nada provechoso al acogido demente la asistencia permanente de un sirviente particular a su lado; pues la experiencia ha demostrado que cuanto más libertad goza este, están tanto más tranquilo, 10 que no sucede cuando siempre tiene a su lado una persona, que o bien le está contradiciendo o excitando su atención aunque sea con buenas y adecuadas palabras, que por 10 regular la mayor parte del tiempo lo pasa en una agitación exacerbada. por estas consideraciones cree esta Dirección que el criado que solicita $D^{a}$ Ana María Alamo del Castillo que sea admitido en esta casa para el servicio particular de su hijo D. Fernando Velasco no solo le es perjudicial a este, sino también a la casa.

Todo 10 que tengo el honor de elevar al superior conocimiento de V.E. en cumplimiento del superior mandato del 21 del actual, acompañando al propio tiempo la solicitud de la interesada como igualmente el informe facultativo sobre el particular afín de que en su vista V.E. resuelva lo que estime más conveniente.

Dios guarde a V.E. m.a.

Leganés 27 de mayo de 1858

Escmo. Sr.

Manuel González

Excmo. Sr. Presidente de la Junta general de Beneficencia del Reino.

Historia 256. Informe del director de la Casa de Dementes de Santa Isabel (Archivo Histórico del Manicomio de Santa Isabel de Leganés, biblioteca del Instituto Psiquiátrico, Servicios de Salud Mental José Germain de Leganés). 